\title{
Magnons dans les super-réseaux ferromagnétiques à 3 - couches
}

\author{
A. AKJOUJ, L. DOBRZYNSKI et B. DJAFARI-ROUHANI
}

Equipe de Dynamique des Interfaces, Laboratoire de Dynamique et Structure des Matériaux Moleculaires, U.R.A. au CNRS n' 801, U.F.R. de Physique, Université de Lille I, 59655 Villeneuve d'Ascq cedex, France

\begin{abstract}
A brief study of bulk and surface magnons in 3-layer Heisenberg ferromagnetic superlattices is presented. Each layer is modelled by a simple-cubic lattice of spins coupled via nearestneighbor exchange interactions. The superlattice has a larger periodicity in the direction perpendicular to the slabs and therefore many magnon branches in the folded Brillouin zone. In the gaps existing between these magnon branches appear the surface-localized magnons. We give closed-form expressions for bulk and surface magnons in 3-layer superlattices, together with the corresponding dispersion curves for one spectific case. An application is also done for a Fe/Co superlattice.
\end{abstract}

Résumé.- Une brève ètude des magnons de volume et de surface dans les super-réseaux à 3-couches est présentée. Chaque couche est modélisée par un réseau cubique simple de spins couplés par des interactions d'échange entre premiers voisins. Le super-réseau possède une grande périodicité dans la direction perpendiculaire aux couches et en conséquence, il existe plusieurs branches de magnons dans la zone de Brillouin réduite. Dans les gaps existants entre ces branches de magnons apparaissent des magnons localisés à la surface. Nous donnerons sous forme analytique les relations de dispersion des magnons de volume et de surface d'un super-réseau à 3 couches que nous illustrons par un exemple. Une application est aussi donnée pour un super-réseau $\mathrm{Fe} / \mathrm{Co}$. 
Les magnons dans les super-réseaux composés de deux couches minces magnétiques différentes ont déjà été étudiés aussí bien expérimentalement que théoriquement [1-4].

Le but de notre papier est d'étudier le cas d'un super-réseau à 3 composants. Ce dernier est obtenu par dépôt alterné et périodique de 3 couches minces de propriétés magnétiques différentes. Nous étudions ce système composite à l'aide du modèle de Heisenberg en limitant les interactions d'échange aux premiers volsins et en négligeant les contributions des énergies dipolaires et Zeeman à l'Hamiltonien.

Notons aussi que, du point de vue mathématique, ce modèle est très similaire à un modèle déjà utilisé pour l'étude des phonons de volume et de surface dans les supers réseaux à $\mathrm{N}$-couches [5].

Ce modèle de magnons a été utilisê auparavant pour les propriétés magnétiques des surfaces [6] et l'étude des magnons localisés dans une lame mince insêrée entre deux autres milieux semi-infinis [7].

Nous utilisons des cristaux cubiques simples avec des interfaces (001) et ayant le même paramètre du réseau ao. Dans chaque cristal, chaque spin $S_{1}$ est couplé avec ses six premiers voisins par les interactions d'échange $J_{1}$.

La relation de dispersion des magnons en volume est donnée par [6]

$$
\omega=4 S_{1} J_{1}\left[3-\cos \left(k_{1} a_{0}\right)-\cos \left(k_{2} a_{0}\right)-\cos \left(k_{3} a_{0}\right)\right]
$$

où $\overrightarrow{\mathbf{k}} \equiv\left(\mathrm{k}_{1}, \mathrm{k}_{2}, \mathrm{k}_{3}\right)$ est le vecteur de propagation.

La fonction réponse de volume correspondante est $[3,6,7]$

$$
G_{01}\left(\ell_{3}, \ell_{3}^{\prime}: k / 1, \omega\right)=\frac{1}{2 s_{1} J_{1}} \frac{t_{1}^{\left|\ell_{3}-\ell_{3}\right|+1}}{t_{1}^{2}-1}
$$

où $\ell_{3}$ donne le numéro des plans atomiques (001),

$$
\vec{k}_{/ /} \equiv\left(\mathbf{k}_{1}, \mathbf{k}_{2}\right) \text { est un vecteur d'onde parallèle aux interfaces, }
$$

$$
\mathbf{t}_{1}=\left\{\begin{array}{lc}
\zeta_{1}-\left(\zeta_{1}^{2}-1\right)^{1 / 2}, & \zeta_{1}>1 \\
\zeta_{1}+1\left(1-\zeta_{1}^{2}\right)^{1 / 2}, & -1<\zeta_{1}<1 \\
\zeta_{1}+\left(\zeta_{1}^{2}-1\right)^{1 / 2}, & \zeta_{1}<-1 .
\end{array} .\right.
$$

et $\quad \zeta_{1}=3-\cos \left(k_{1} a_{0}\right)-\cos \left(k_{2} a_{0}\right)-\frac{\omega}{4 S_{1} J_{1}}$.

Dans la suite, nous utlliserons $t_{1}$ sous la forme $t_{1}=e^{q i}$.

On appellera $J_{11}$, l'interaction d'échange à l'interface entre les lames de type 1 et $i^{\prime}$.

La relation de dispersion des magnons dans le super-réseau à 3-couches apparaît au dénominateur de la fonction réponse $g\left(n^{\prime} \ell_{3}: n^{\prime} i^{\prime} \ell^{\prime}{ }_{3}\right)$ que nous calculons en utilisant la théorie des réponses d'interface [8]. Dans ce papier, nous donnerons ces expressions pour un super-réseau à trois couches. Pour les magnons de volume : 
$2 \cos \left(k_{3} D\right)=2 B_{1} B_{2} B_{3}+\frac{C_{1} C_{2} C_{3}}{J_{12} J_{23} J_{31}}+\varepsilon_{1 j k}\left\{B_{i} A_{j} C_{k}+\frac{1}{2} B_{1} B_{j} C_{k}\left(\frac{1}{J_{12}}+\right.\right.$

$$
\left.\left.\frac{1}{J_{23}}+\frac{1}{J_{31}}\right)+\frac{1}{2 J_{j k}} A_{1} C_{j} C_{k}+\frac{1}{2 J_{j k}} B_{1} C_{j} C_{k}\left(\frac{1}{J_{k 1}}+\frac{1}{J_{1 j}}\right)\right\}
$$

où $\quad D=a_{0} \sum_{i=1}^{3} L_{i}$ est la période du super-réseau,

$\varepsilon_{i j k}=\left\{\begin{array}{cc}1 & \text { si } i, j, k \text { est une permutation directe de } i, j, k=1,2,3 \\ -1 & \text { si } i, j, k \text { est une permutation indirecte de } i, j, k=1,2,3 \\ 0 & \text { autrement, }\end{array}\right.$

$A_{i}=\frac{1}{J_{1}} \frac{\operatorname{sh}\left[\left(L_{i}-1\right) q_{i}\right]}{\operatorname{sh}\left(q_{i}\right)}, \quad B_{1}=\frac{\operatorname{ch}\left[\left(L_{i}-1 / 2\right) q_{1}\right]}{\operatorname{ch}\left(q_{i} / 2\right)}, \quad C_{i}=2 J_{i}$ th $\left(q_{i} / 2\right) \operatorname{sh}\left(q_{i} L_{i}\right)$

L'équation (5) peut être résolue numériquement. Dans la fig. 1, nous présentons les résultats de la structure de bande du super-réseau dans le cas où $L_{1}=L_{2}=L_{3}=2$. Les bandes de volume sont représentées par les aires hachurées. Celles-ci sont séparées par des gaps où peuvent exister les magnons de surface que nous discuterons ci-dessous.

Pour obtenir un super réseau avec une surface libre, il faut cliver le super-réseau infini par l'élimination de toutes les interactions entre deux lames voisines. La fonction réponse $\stackrel{\leftrightarrow}{g}_{s}$ du super-réseau semi-infini nécessite la connaissance de la fonction réponse $\overleftrightarrow{\mathrm{g}}$ du super-réseau infini. Pour l'étude des modes localisés à la surface, nous n'avons besoin que de l'élément de $\overleftrightarrow{\mathrm{g}}_{\mathrm{s}}$ sur le site de surface. Ainsi, pour le super-réseau à 3-couches semi-infini ayant la couche de type 1 à la surface suivie de la couche de type 2 puis 3 et ainsi de suite, nous obtenons, en utilisant la théorie des réponses d'interface [8],

$$
g_{s}(1 \quad 1 \quad 1: 1 ; 11)=-\frac{A_{s}(\omega)-t}{D(\omega)}
$$

où $\quad A_{s}(\omega)=B_{1} B_{2} B_{3}+A_{1} C_{2} B_{3}+A_{1} B_{2} C_{3}+B_{1} A_{2} C_{3}+\frac{1}{J_{23}}\left(A_{1} C_{2} C_{3}+B_{1} B_{2} C_{3}\right)$

$$
+\frac{1}{J_{12}}\left(B_{1} C_{2} B_{3}+B_{1} B_{2} C_{3}\right)+\frac{B_{1} C_{2} C_{3}}{J_{12} J_{23}}
$$




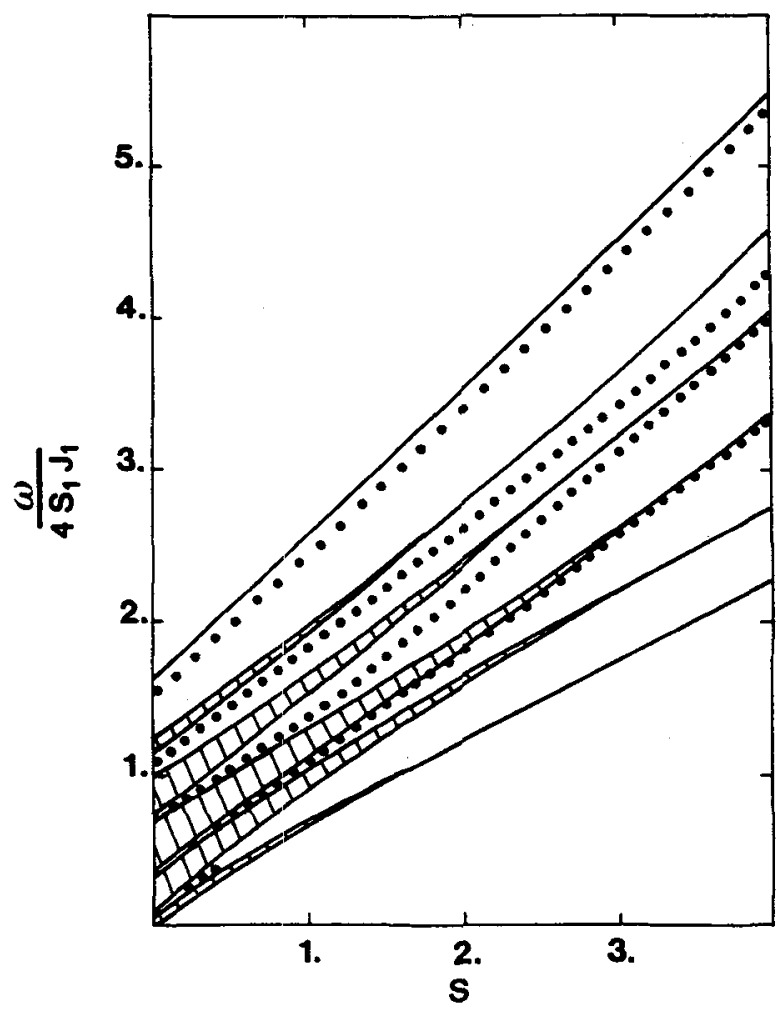

Fig. 1.- Structure de bande des magnons $\frac{\omega}{4 \mathrm{~S}_{1} \mathrm{~J}_{1}}$ pour un super-réseau à 3 couches, avec $\mathrm{L}_{1}=\mathrm{L}_{2}=\mathrm{L}_{3}=2$, en fonction du paramétre $\mathrm{S}=2$ - $\cos \left(\mathrm{k}_{1} \mathrm{a}_{0}\right)$ $\cos \left(k_{2} \mathrm{a}_{0}\right)$. Les intéractions d'échanges sont définies par $J_{3} / J_{1}=0.5$; $\mathrm{J}_{2} / \mathrm{J}_{1}=\mathrm{J}_{31} / \mathrm{J}_{1}=0.75 ; \mathrm{J}_{12} / \mathrm{J}_{1}=0.875$ et $\mathrm{J}_{23} / \mathrm{J}_{1}=0.675$. Les aires hachurées présentent les bandes de volume et les pointillés présentent les courbes de dispersion des magnons de surface lorsque le film $\mathbf{i}=1$ est à la surface.

et $D(\omega)=B_{1} B_{2} C_{3}+B_{1} C_{2} B_{3}+C_{1} B_{2} B_{3}+C_{1} A_{2} C_{3}+C_{1} A_{2} C_{3}$

$$
+\frac{1}{J_{23}}\left(C_{1} B_{2} C_{3}+B_{1} C_{2} C_{3}\right)+\frac{1}{J_{12}}\left(C_{1} C_{2} B_{3}+C_{1} B_{2} C_{3}\right)+\frac{C_{1} C_{2} C_{3}}{J_{12} J_{23}}
$$

Les fréquences $\omega$ des modes localisés à la surface sont données par

$$
D(\omega)=0
$$

avec la condition, [9]

$$
\left|A_{s}(\omega)\right|>1
$$

qui assure que l'amplitude de ces modes décroit en pénétrant à l'intérieur du super-réseau.

Les modes de surface dépendent de la nature de la couche qui est en surface, de son épaisseur (qui peut être supposée différente de celles des couches dans le volume du super-réseau), ainsi que de l'ordre des différents matériaux à partir de la surface (par exemple $1 / 2 / 3 \ldots$ ou $1 / 3 / 2$... etc...). 
Sur l'exemple de la fig. 1 nous avons représenté ces modes de surface dans le cas où les matériaux sont disposés dans l'ordre $1 / 2 / 3 \ldots$; dans ce cas quatre branches de modes de surface apparaissent dans la majeure partie de la zone de Brillouin. Sur la fig. 2 nous avons aussi illustré l'exemple plus simple d'un super-réseau à deux constituants $\mathrm{Fe}$ Co avec $\mathrm{L}_{\mathrm{Fe}}=2$ et $\mathrm{L}_{\mathrm{Co}}=4$; les modes de surface sont représentés selon que le super-réseau se termine par un film de $\mathrm{Fe}$ ou un film de Co. Les résultats de ce dernier cas sont qualitativement similaires à ceux de la fig. 1 , tandis que les résultats lorsqu'un film de Fe est en surface ressemblent à ceux pour le super-réseau à trois constituants avec l'ordre $3 / 2 / 1 \ldots$ des

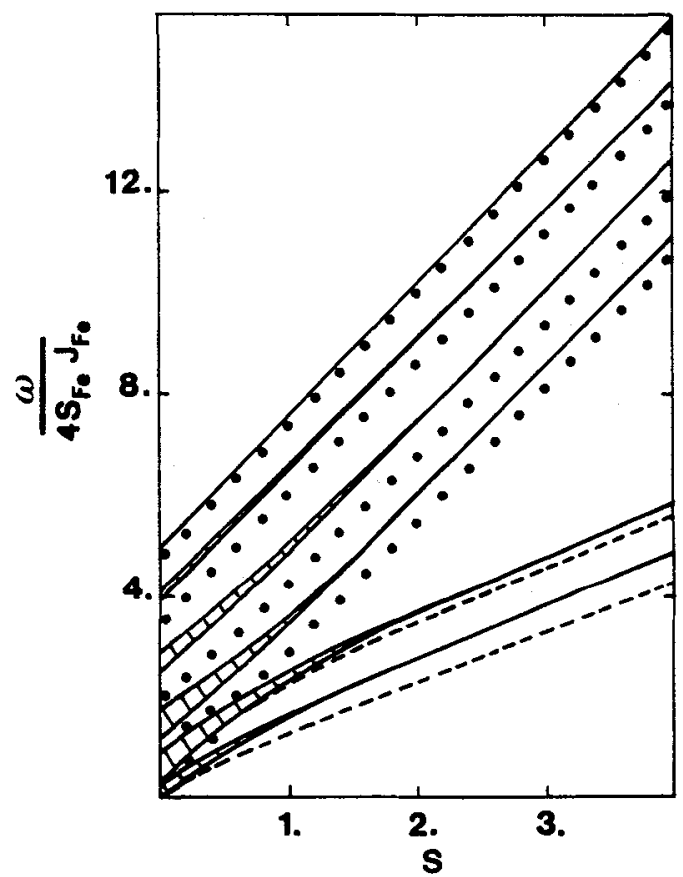

Fig 2.- Magnons de volume (aires hachurees) et de surface dans un superréseau $\mathrm{Fe}-\mathrm{Co}_{0}, \mathrm{~L}_{\mathrm{Fe}}=2$ et $\mathrm{L}_{\mathrm{Co}}=4$, lorsque $\mathrm{Fe}(--)$ ou $\mathrm{Co}\left({ }^{\circ}\right.$. $)$ est à la surface. $\mathrm{S}_{\mathrm{Co}} / \mathrm{S}_{\mathrm{Fe}}=0.72 ; \mathrm{J}_{\mathrm{Co}} / \mathrm{J}_{\mathrm{Fe}}=3.64$ et $\mathrm{J}_{\mathrm{Fe}-\mathrm{Co}} / \mathrm{J}_{\mathrm{Fe}}=2.9$.

matériaux à partir de la surface. Cependant lorsqu'on considère le superréseau à trois composants avec les autres ordres possibles des matériaux. les branches de modes de surface sont differrentes de celles sur la fig. 2 , notamment en ce qui concerne la disposition de ces branches par rapport aux branches de volume. Remarquons que nous obtenons deux branches de modes de surface pour les ordres $1 / 3 / 2 \ldots$ et $3 / 1 / 2 \ldots$ et quatre branches pour les ordres $2 / 3 / 1 \ldots$ et $2 / 1 / 3 \ldots$; dans ce dernier cas, l'une des branches croise une bande de volume.

Globalement, on aboutit à une variété plus importante de modes caractéristiques de la surface dans le cas du super-réseau à trois composants par rapport à celui du super-réseau à deux constituants.

Enfin, notons que des résultats encore différents peuvent s'obtenir si la couche de surface a une épaisseur plus petite ou plus grande que celles des couches dans le volume du super-réseau. 
En conclusion, nous avons obtenu pour la première fois les magnons de volume et de surface pour un super-réseau ferromagnétique composé de 3 constituants dans le modèle de Heisenberg. La simplicité de ce modèle nous permet de déterminer sous forme analytique les fonctions réponse pour le super-réseau. Les pôles de ces fonctions réponse donnent les relations de dispersion des magnons de volume et de surface des super-réseaux. Signalons ëgalement que notre calcul peut s'appliquer au cas des super-rêseaux avec des couplages antiferromagnétiques aux interfaces [10]. Cette théorie peut aussi être complétée dans l'avenir en tenant compte des contributions des énergies dipolaires et Zeeman [11].

\section{Rêférences}

[1] T. Jarlborg and A.J. Freeman, J. Appl. Phys. 53, 8041 (1982).

T. Shinjo, K. Kawaguchi, R. Yamamoto, N. Hosaito and T. Takada, J. Phys. (Paris) Colloq. 45, C5-367 (1984) ; N.K. Flevaris, J.B. Kelterson and J.E. Hilliard, J. Appl. Phys. 53, 8046 (1982).

[2] R.E. Camley, T.S. Rahman and D.L. Mills, Phys. Rev. B27, 261 (1983) ; F. Herman, P. Lambin and D. Jepsen, Phys. Rev. B31, 4394 (1985) ; K. Mika and P. Grünberg, Phys. Rev. B31, 4465 (1985).

[3] L. Dobrzynski, B. Djafari-Rouhani and H. Puszkarski, Phys. Rev. B32, 3251 (1986).

[4] E.L. Albuquerque, P. Fulco, E.F. Sarmento and D.R. Tilley, Solid State Comm. 58, 41 (1986).

[5] T. Szwacka, A. Noguera, A. Rodriguez, J. Mendialdua and L. Dobrzynski, Phys. Rev. B37, 8451 (1988) ; Phys. Rev. B39, 12568 (1989).

[6] L. Dobrzynski and D.L. Mills, Phys. Rev. B186, 538 (1969).

[7] A. Akjouj, B. Sylla, P. Zielinski and L. Dobrzynski, Phys. Rev. B37, 5670 (1988).

[8] L. Dobrzynski, Prog. in Surf. Sci., 26, 103 (1987).

[9] L. Dobrzynski, B. Djafari Rouhani, O. Hardouin Duparc, Phys. Rev. B29, 3138 (1984).

[10] A. Akjouj, B. Djafari-Rouhani, L. Dobrzynski and H. Puszkarski, to be published

[11] R.E. Camley, T.S. Rahman and D.L. Mills, Phys. Rev. B27, 261 (1983). 